\title{
Dynamic and three-dimensional transcranial sonography studies of an asymptomatic, cerebral convexity arachnoid cyst
}

\section{Case report}

Felix Schlachetzki, M.D., Thilo Hölscher, M.D., O.W. Ullrich, M.D. Sabine Kübber, M.D., Wendelin Blersch, M.D., Birgit Götz, M.Sc., Katrin Ocklenburg, M.Sc., Berthold Schalke, M.D., and Ulrich Bogdahn, M.D.

Departments of Neurology and Neurosurgery, University of Regensburg, Regensburg, Germany; Department of Pediatric Psychiatry, Bezirksklinikum Regensburg, Regensburg, Germany; and EchoTech 3D Imaging Systems GmbH, Halbergmoos, Germany

Dynamic and three-dimensional transcranial sonography (dTCS and 3D-TCCS) examinations are complementary, noninvasive methods used in the assessment and follow up of patients with cerebrospinal fluid (CSF) circulation disorders.

A 16-year-old female patient who presented with a space-occupying, cerebral convexity arachnoid cyst and recurrent tension-type headache underwent examination for raised intracranial pressure (ICP) by using a standard color-coded duplex sonography system attached to a personal computer--based system for 3D data acquisition. Conventional TCS identified the outer arachnoid membrane of the cyst, which undulated freely after short rotation of the head ("headshake maneuver"). The undulation was documented as a QuickTime movie that is included with this article. A 3D dataset was acquired and, by using a multiplanar reformatting reconstruction algorithm, the authors obtained images with excellent resolution that corresponded to an initial magnetic resonance (MR) imaging study. No differences on dTCS and 3D-TCS were detectable at follow up 9 months later, indicating normal ICP and a stable, benign condition.

The use of 3D-TCCS and dTCS ultrasonography may complement other diagnostic procedures such as MR imaging and, thus, can improve management and therapeutic strategies for patients with CSF circulation disorders. In this report the authors present evidence of the excellent fine resolution and exact reproducibility of reconstructed ultrasound image planes derived from 3D datasets and the additional biomedical information from dynamic imaging.

Key Words * transcranial ultrasonography * three-dimensional sonography * arachnoid cyst * dynamic sonography * raised intracranial pressure * brain imaging * cerebrospinal fluid disorder 
Since the first descriptions of ultrasound brain images obtained through the temporal squama,[11,19] transcranial sonography (TCS) and transcranial color-coded duplex sonography (TCCS) have expanded the range of application in diagnostic ultrasonography of the central nervous system.[3] The "real-time" character of these methods allow for dynamic studies, and in 1994, Becker, et al.,[2] demonstrated, through the use of dynamic TCS (dTCS), a reduced undulation capacity of the septum pellucidum after mild shaking of the head in patients with raised intracranial pressure (ICP); however, dTCS tests for the evaluation of raised ICP in cerebrospinal fluid (CSF) circulation disorders have limited sensitivity and specificity. Ultrasonography, by nature, is still a highly investigator-dependent diagnostic technique. Important limitations of TC ultrasonography imaging are addressed by three-dimensional (3D) methods that promise reduced investigator dependency, improved reproducibility, quantitative depiction of brain structures, and thus, are well suited for repeated (retrospective and prospective) investigations.[6, 8]

In this study, we present a young patient with a space-occupying cerebral convexity arachnoid cyst and with hypogenesis of the temporal lobe. Dynamic TCS for the assessment of changes in ICP and 3D-TCCS image--reconstruction techniques were performed at initial assessment and at a follow-up examination 9 months later.

\section{CASE REPORT}

Presentation and Examination. A 16-year-old girl was referred to our clinic for neurological evaluation of bifrontal chronic headache and vertigo, as well as lack of concentration. Prior to consultation, magnetic resonance (MR) imaging performed after the patient had been sedated revealed a space-occupying cerebral convexity arachnoid cyst of the right hemisphere and hypogenesis of the ipsilateral temporal lobe. Her neurological status was completely normal. On psychiatric examination, higher cognitive functions were limited, suggestive of slight mental retardation, although no intelligence or projective tests could be performed because of noncompliance. The psychiatric diagnosis was an adjustment disorder with mixed emotional features (DSM III 309.28) in stress situations at school. In addition, there was a history of child abuse in her early youth. After 9 months, she was readmitted for recurrent symptoms; again, the neurological examination was unremarkable. On both occasions, the patient was referred to our ultrasonography laboratory.

Transcranial Sonography Examinations. Transcranial sonography examinations were performed using a conventional color-coded duplex ultrasound system, equipped with a 2 to $3.5--\mathrm{MHz}$ phase array transducer (Sequoia 512, 3V2c transducer, Acuson, Mountain View, CA). Three-dimensional data acquisition was achieved using a personal computer--based free-hand system, consisting of an electromagnetic field transmitter and a receiver (3D-FreeScan; EchoTech, Munich, Germany).

Transcranial sonography was performed with a transmission frequency of $3.5 \mathrm{MHz}$, dynamic range of 80 $\mathrm{dB}$, and the lowest persistence possible. Parameters remained unchanged at the 9-month follow-up examination.

For dTCS studies, the transducer was firmly pressed on the left temporal acoustic bone window, and the head of the patient was slightly shaken at a frequency of approximately 3 to $4 \mathrm{~Hz}$ ("headshake maneuver"). The resulting images were stored as an MPEG movie sequence of approximately 4 seconds.

For 3D data acquisition, the field transmitter was placed around the patient's head, producing a voxel-specific magnetic field. The receiver (position sensor), to determine orientation within a hemisphere of $1 \mathrm{~m}$ around the transmitter, was attached to the transducer. While tilting the transducer at 
the left temporal acoustic bone window, images of the duplex system were retrieved from the color video signal output, digitized, and stored together with data obtained on the position and orientation in space from the magnetic field sensor. By using a technique called multiplanar reformatting, a 3D voxel-based image was reconstructed and presented as a polyhedron with three perpendicular image planes. To view other planes, the axial-oriented planes were moved through the volume to match the corresponding $\mathrm{T}_{1}$-weighted MR image plane. For a more detailed description, refer to the study by Fenster.[8]

Transcranial Sonography Finding. Orientation in TCS through both the temporal acoustic bone windows was difficult due to the shifted intracranial anatomy. When the hypoechogenic mesencephalic brainstem was seen to insonate through the left acoustic bone window, we identified a large hypoechogenic, frontoparietal convexity lesion and a highly echogenic septum arising from the rudimentary right temporal lobe and upper diencephalon, presumably the outer arachnoid membrane. This membrane was not seen on an MR imaging study. When the headshake maneuver was instigated, a smooth undulation of the membrane was induced that lasted up to 2 seconds after the procedure. At 9-month follow-up examination, no change of the undulation capacity was observed (Fig. 1), as analyzed by QuickTime movie documentation.

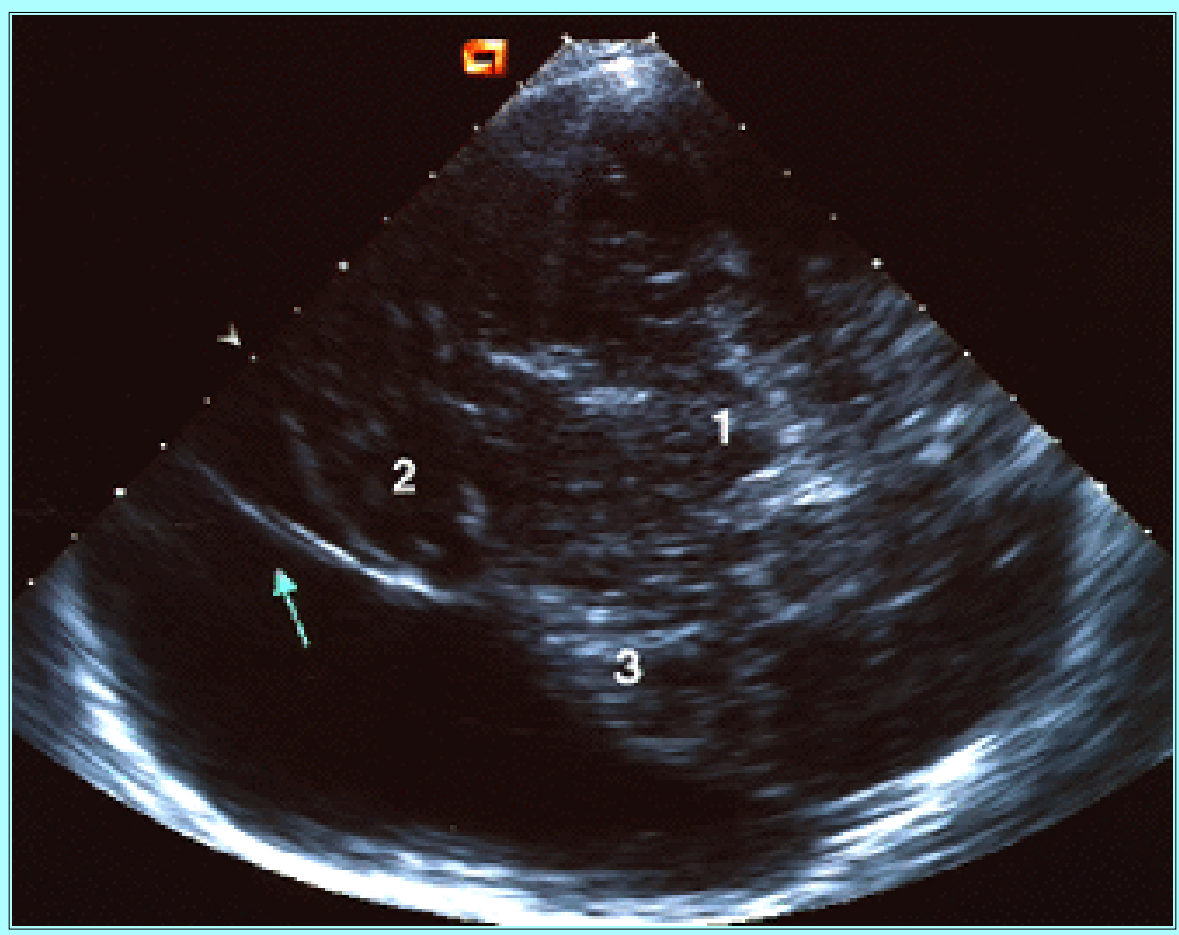

Fig. 1. Transcranial sonography B-mode image obtained through the left temporal acoustic window: 1) hypoechogenic mesencephalic brainstem with the surrounding echogenic basal cisterns; 2) hypoechogenic right frontal lobe; 3 ) rudimentary right temporal lobe; white arrow marks the outer membrane within the enlarged arachnoid cyst.

Click here to view QuickTime movie in which the membrane indicated in Fig. 1 undulates freely when the patient's head is lightly rotated ("headshake maneuver").

\section{Download QuickTime}

Three-dimensional voxel-based TCS datasets were obtained through the right acoustic temporal bone window. Reconstructed images at the level of the basal ganglia, according to the MR image plane (Fig. 2 left), were calculated. The new two-dimentional ultrasound images were of high resolution: the falx cerebri, the contralateral insula, both choroid plexus, the rudimentary temporal lobe, and even different 
thalami nuclei could be assessed. The septum, which had already been identified on conventional TCS, could also be identified and was absent on MR imaging. At follow up, no change could be observed, demonstrating very good reproducibility (Fig. 2 right).
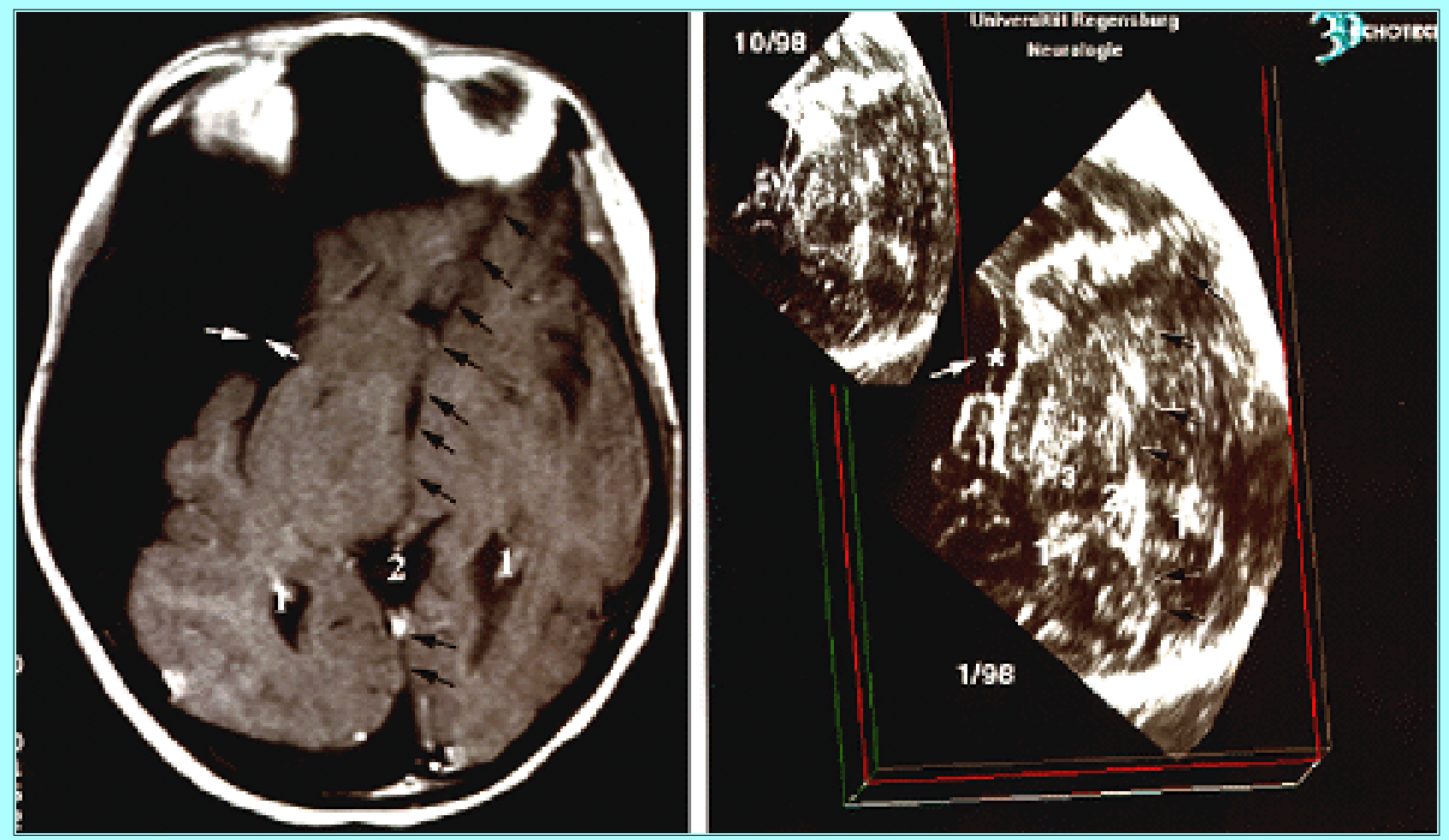

Fig. 2. Left: $A T_{1}$-weighted MR image obtained at the level of the basal ganglia: 1) choroid plexus; 2) pineal gland; white arrows indicate the location of the outer arachnoid membrane as detected by ultrasonography; black arrows mark the shifted midline. Right:

Two-dimensional ultrasound reconstructions of the 3D dataset: 1) choroid plexus; 2) pineal gland (echogenic); 3) the lateral thalmic nuclei are slightly more echogenic than the medial thamic nuclei located between 2 and 3; asterisk and the white arrow mark the outer arachnoid membrane; black arrows point at the shifted midline (third ventricle, falx cerebri). Image in upper left corner: Two-dimensional image reconstruction obtained 9 months later, demonstrating an unchanged, stable condition.

\section{DISCUSSION}

The results of our report illustrate the potential utility of 3D-TCS and dTCS in aiding the examination of a patient with CSF circulation disorder. Specifically we demonstrate the highly improved resolution of the reconstructed two-dimensional ultrasound image planes over conventional ultrasound images, the excellent reproducibility during follow-up examination because of 3D dataset analysis, and finally, the advantage of dynamic, real-time ultrasonography.

The use of TCCC in the diagnosis of vascular and parenchymal disease has been previously described by a number of authors. $[3,4,11,13,15,20]$ Since the early 1980 s obtaining TCS images through the open anterior fontanelle has been the primary diagnostic procedure for pediatric patients, yielding excellent results. $[1,5,10,14,17,21,22]$ Noninvasive assessment of CSF disorders in the elderly is performed using cerebral computerized tomography (CT) and MR imaging; however, with more advanced ultrasound equipment, assessment of the third ventricle, the lateral ventricles, and a midline shift has become feasible even in patients with insufficient acoustic bone windows. Results of evaluation of the third 
ventricle, at an insonation angle of 80 to $90^{\circ}$, are superior to those of the lateral ventricle, and the technique allows for the estimation of a potential midline shift with great accuracy in cases such as those with space-occupying hemispheric infarction. [2,12]

In 1994, dTCS was used to assess raised ICP. In a study of 26 patients, Becker, et al.,[2] found undulating septa pellucida and CSF pressures below $25 \mathrm{~cm} \mathrm{H}_{2} \mathrm{O}$. In terms of raised ICP and ventricular dilation, the septum is tight, thus no movement may be observed. However, with this method, two false-negative diagnoses were reached: one in a patient with meningitis and one in a patient with dilated ventricles (normal-pressure hydrocephalus), limiting the sensitivity and specificity.[2] The flexibility of this method allows for its use as a bedside and noninvasive procedure, even within the intensive care unit.

Arachnoid cysts are incidental findings in adults, sometimes leading to complications after head injury. In children a cyst is diagnosed when it the cyst rapidly enlarges and becomes symptomatic, interfering with adjacent neural structures or obstructing the CSF circulation. The term "primary congenital arachnoid cysts" is applied to cases in which there are collections of fluid that develop within the arachnoid membrane due to splitting or duplication of this structure. Arachnoid cysts overlying most or all of the surface of one hemisphere, with agenesis or hypogenesis of the temporal lobe, vary in their clinical manifestation.[7] Both CT and MR imaging often fail to detect the outer or inner arachnoid membrane.[18] The best neurosurgical therapy, such as excision of the outer membrane in noncommunicating arachnoid cysts, is a matter of current discussion and beyond the scope of this article.

In this case, we took advantage of the undulation capacity of the long echogenic outer arachnoid membrane within the subarachnoid cyst for assessment of raised ICP and follow-up examination. The membrane undulated well, indicating a pressure equilibrium in the CSF compartments and, thus, no pressure-active cyst. Qualitative comparison of the QuickTime movie documentation revealed no difference 9 months later.

Three-dimensional reconstruction has become feasible with advances in computer technology in which conventional ultrasound equipment is used with a personal computer--based mechanical or free-hand system (magnetic field positioners).[6] Three-dimensional sonography can be used to address some limitations of sonography: by creating a 3D dataset, repeated (retrospective and prospective) investigations with reduced investigator dependence and quantitative depiction of brain structures may become feasible. Thus far, TCS and TCCS are limited to angulated, not standardized, image planes by the physics of the temporal acoustic bone window; this may also be overcome in 3D image reconstruction. $[6,8,9,16]$ Multiplanar reformatting is a reconstruction algorithm in which the whole voxel-based 3D dataset is used to create new image planes and, thus, allows for reconstruction of standard, axial-oriented, canthomeatal-oriented image planes derived from cerebral CT and MR imaging. Other limitations, such as the inferior ultrasound near-field and the sector format, arise from the low-frequency phase array transducer and allow for parenchymal imaging of mainly the midline structures and the contralateral hemisphere.[3,19] Thus, for a more complete assessment of the outer arachnoid membrane on the right side, the left temporal acoustic bone window was selected. The 3D reconstructions focus on the midline and preserved left hemisphere; consequently, insonation was performed from the right side. However, the hypogenic right temporal lobe could still be assessed from the ipsilateral side in this case.

In this study, 3D-TCCS, new and highly accurate images correlating to the initial MR image planes, were 
reconstructed from datasets. The images stress the ventricular borders, the margin of the compressed temporal lobe, the location of the membrane, and the midline structures. Because these new images were of a very high resolution, they allowed even the differentiation of the ipsilateral lateral and the medial thalami nuclei. In the follow-up examination, this image plane could be reconstructed with great accuracy, demonstrating that $3 \mathrm{D}$ data reconstruction of ultrasound images is a highly reproducible technique suited for follow-up examination.

The clinical presentation of the cerebral convexity arachnoid cyst, the unchanged undulation capacity of the outer arachnoid membrane in dTCS and the 3D-TCS studies, with their good anatomical correlation to the initial MR image and their very good reproducibility, were not suggestive of a pressure-active CSF circulation disorder; thus, invasive ICP measurements were not performed.

\section{CONCLUSIONS}

Dynamic TCS and 3D-TCS studies are modern tools for brain imaging that allow for the qualitative assessment of CSF circulation disorders. Although these methods cannot replace cerebral CT and MR imaging as primary diagnostic procedures, they may prevent the use of additional invasive tests and, once the diagnosis is established, aid in the temporary management of patients with CSF circulation disorders. Three-dimensional reconstruction is superior to conventional TCS and yields excellent morphological images and high reproducibility. The advantages of TCS as a noninvasive and mobile tool without inherent risk for the patient allow for the application not only in pedriatic neuroimaging but also in the intensive care unit.

\section{References}

1. Babcock DS, Han BK: The accuracy of high resolution, real-time ultrasonography of the head in infancy. Radiology 139:665-676, 1981

2. Becker G, Bogdahn U, Strassburg HM, et al: Identification of ventricular enlargement and estimation of ventricular pressure by transcranial color-coded real-time sonography. J Neuroimaging 4:17-22, 1994

3. Bogdahn U, Becker G, Schlachetzki F (eds): Echoenhancers and Transcranial Color Duplex Sonography. Berlin: Blackwell, 1998

4. Bogdahn U, Becker G, Winkler J, et al: Transcranial color-coded real-time sonography in adults. Stroke 21:1680-1688, 1990

5. Cooke RWI: Ultrasound examination of neonatal heads. Lancet 2:38, 1979 (Letter)

6. Delcker A, Knappertz VA, Wilson J, et al: Transcranial 3D B-mode imaging in a case of cerebral hemorrhage. J Neuroimaging 8:173-175, 1998

7. Di Rocco C: Arachnoid cysts, in Youmans JR (ed): Neurological Surgery, ed 4. Philadelphia: WB Saunders, 1996, Vol 2, pp 967-994

8. Fenster A: 3-D sonography--technical aspects, in Bogdahn U, Becker G, Schlachetzki F (eds): Echoenhancers and Transcranial Color Duplex Sonography. Berlin: Blackwell, 1998, pp 121-140

9. Fenster A, Downey DB: 3-D ultrasound imaging: a review. IEEE Eng Med Biol 15:41-51, 1996 
10. Fischer AQ, Livingstone JN II: Transcranial Doppler and real-time cranial sonography in neonatal hydrocephalus. J Child Neurol 4:64-69, 1989

11. Furuhata H: Historical development of transcranial color-coded tomography, in Bogdahn U, Becker G, Schlachetzki F (eds): Echoenhancers and Transcranial Color Duplex Sonography. Berlin: Blackwell, 1998, pp 3-15

12. Gerriets T, Stoltz E, Modrau B, et al: Sonographic monitoring of midline shift in hemispheric infarctions. Neurology 52:45-49, 1999

13. Goertler M, Kross R, Baeumer M, Diagnostic impact and prognostic relevance of early contrast-enhanced transcranial color-coded duplex sonography in acute stroke. Stroke 29:955-962, 1998

14. Grant EG, White EM: Pediatric neurosonography. J Child Neurol 1:319-337, 1986

15. Lindner A, Becker G, Bogdahn U, et al: [Evaluation of transcranial color-coded-real-time-sonography in the diagnosis of cerebral lesions.] Nervenarzt 66:252-260, 1995 (Ger)

16. Lyden PD, Nelson TR: Visualization of the cerebral circulation using three-dimensional transcranial power Doppler ultrasound imaging. J Neuroimaging 7:35-39, 1997

17. Pape KE, Blackwell RJ, Cusick G, et al: Ultrasound detection of brain damage in preterm infants. Lancet 1:1261-1264, 1979

18. Rengachary SS, Kennedy JD: Intracranial arachnoid and ependymal cysts, in Wilkins RH, Rengachary SS (eds): Neurosurgery, ed 2. New York: McGraw-Hill, 1996, Vol 3, pp 3709-3728

19. Schöning MD, Grunert D, Stier B: [Transcranial real-time sonography in children and adolescents, ultrasound anatomy of the brain.] Ultraschall Med 9:286-292, 1988 (Ger)

20. Seidel G, Kaps M, Dorndorf W: Transcranial color-coded duplex sonography of intracranial hematomas in adults. Stroke 24:1519-1527, 1993

21. Shackelford GD: Neurosonography of hydrocephalus in infants. Neuroradiology 28:452-462, 1986

22. Timor-Tritsch IE, Monteagudo A, Cohen HL (eds): Ultrasonography of the Prenatal and Neonatal Brain. Stamford, Conn: Appleton \& Lange, 1996

Manuscript received October 27, 1999.

Accepted in final form November 30, 1999.

Address reprint requests to: Felix Schlachetzki, M.D., Department of Neurology, University of Regensburg, Bezirksklinikum Regensburg, Universitätsstrasse 84, 93053 Regensburg, Germany. e-mail: felix.schlachetzki@klinik.uni-regensburg.de. 